\title{
ON HALPERN'S CONJECTURE FOR CLOSED PLANE CURVES
}

\author{
TETSUYA OZAWA
}

\begin{abstract}
Let $c$ be a smooth closed plane curve given in general position. A bitangent of $c$ is, by definition, a line which is tangent to $c$ at two different points. Let $B(c)$ and $D(c)$ denote the numbers of all bitangents and all double points of $c$, respectively. We prove here that if $c$ has no inflection points, $B(c) \leq D(c)(2 D(c)-1)$. This is the affirmative answer to Halpern's conjecture.
\end{abstract}

0. Let $c: S^{1} \rightarrow \mathbf{R}^{2}$ be a smooth closed curve. If $c$ is in general position, then $c$ has a finite number $B(c)$ of bitangents, that is, lines in $\mathbf{R}^{2}$ which are tangent to $c$ at two points. A bitangent $T$ is said to be exterior or interior, according as in small neighborhoods of the two tangent points of $T$ to $c$, the curve $c$ lies on the same side or on opposite sides of $T$. Let $I I(c)$ and $I(c)$ denote the numbers of exterior and interior bitangents of $c$, respectively; $B(c)=I I(c)+I(c)$. We consider other two geometric objects; the double points and the inflection points. A smooth plane curve $c$ has generically a finite number $D(c)$ of double points and no triple points, nor more, and a finite number $F(c)$ of inflection points. It is clear that $F(c)$ is even. (See Figure 1.)

Fabricius-Bjerre discovered an equation which $I I(c), I(c), D(c)$ and $F(c)$ obey;

$$
I I(c)=I(c)+D(c)+F(c) / 2 .
$$

This equation is a necessary, but not sufficient, condition in the following sense; for example, if $D(c)=F(c)=0$, then $c$ is convex, hence $B(c)=I I(c)+I(c)=0$; in other words, $(I I, I, D, F / 2)=(1,1,0,0)$ satisfies $(1)$ but cannot be realized as

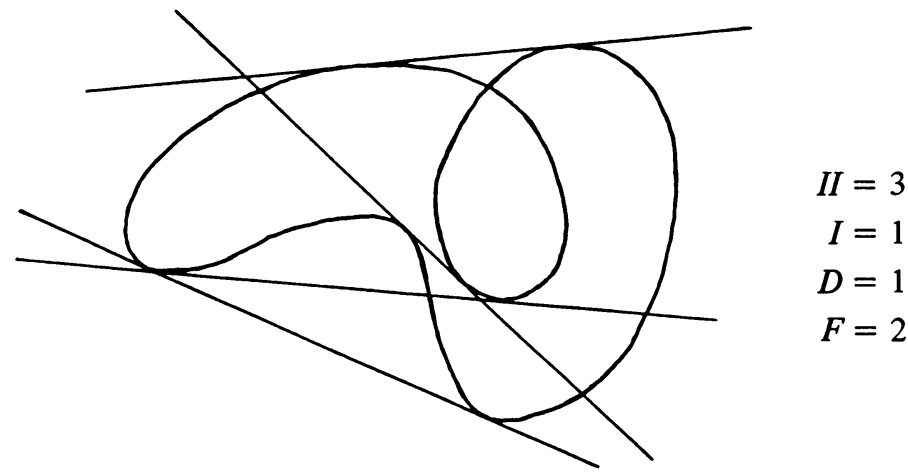

FIGURE 1

Received by the editors December 17, 1983.

1980 Mathematics Subject Classification. Primary 53A04.

Key words and phrases. Closed plane curve, bitangent, double point, inflection point, tangential degree.

(C) 1984 American Mathematical Society $0002-9939 / 84 \$ 1.00+\$ .25$ per page 


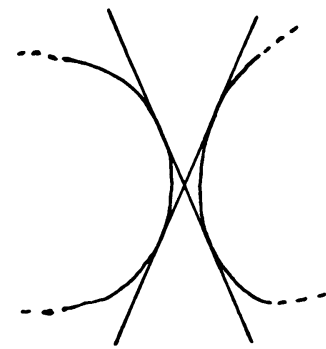

General position

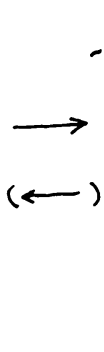

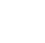

Special position

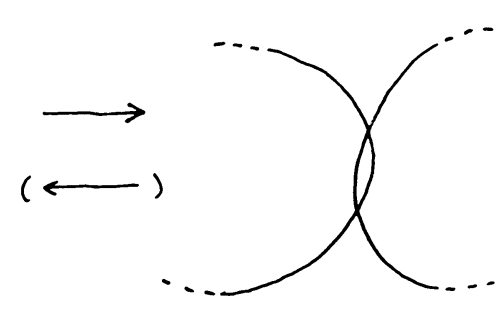

General position

FIGURE 2

$(I I, I, D, F / 2)=(I I(c), I(c), D(c), F(c) / 2)$ by some curve $c$. The question is the following;

Question. What is the necessary and sufficient condition for integers $I I, I, D$, and $F / 2$ to be attained by the integers $I I(c), I(c), D(c)$ and $F(c) / 2$ for some curve $c$ in general position.

Halpern gave this question in $[\mathbf{3}]$ and conjectured that:

(i) any quadruplet $(I I, I, D, F / 2)$ satisfying (1) with $F / 2 \geq 1$ can be attained by a curve;

(ii) a quadruplet $(I I, I, D, F / 2)$ with $F=0$ should satisfy the inequality $I \leq$ $D^{2}-D$ and the equality $I \equiv 0(\bmod 2)$ besides $(1)$ in order to be attained by a curve.

In that paper Halpern mentioned that general examples show that (i) is true. We will see this in $\S 3$. He also said it can be shown that, if $F(c)=0, I(c)$ is even and $I(c) \leq 4 D^{2}(c)+2 D(c)$. The first part is proved by using a deformation argument in the sense of Banchoff (cf. [1]), as follows: let $c$ be a given curve with $F(c)=0$, and let $d(c)$ denote the tangential degree of $c$. There is a curve $c_{0}$ such that $F\left(c_{0}\right)=0, d\left(c_{0}\right)=d(c)$, and $I\left(c_{0}\right)$ is even (for example, the curve $c(l, m, n$ ) with $l=m=0$ and $n=|d(c)|=1$, defined in $\S 3$, Figure 7 , has an even number of interior bitangents; in fact, $I(c(0,0, n))=n(n-1)$. $c_{0}$ can be deformed to $c$ preserving $F=0$ in such a controlled way that the changes of $I$ at the special position in the deformation can be observed. The only special position for which the integer $I$ will change is as in Figure 2. The interior bitangents appear or disappear in pairs. It follows that $I(c)$ is even since $I\left(c_{0}\right)$ is even.

Therefore the main part of the conjecture is, if $F(c)=0, I(c) \leq D^{2}(c)-D(c)$; in other words, if a smooth closed plane curve $c$ in general position has no inflection points, then the number $B(c)$ of bitangents is less than or equal to $2 D^{2}(c)-D(c)$, where $D(c)$ denotes the number of double points.

The purpose of this note is to show that Halpern's conjecture is true, and to answer the above question in the

THEOREM. The necessary and sufficient condition for nonnegative integers $I I$, $I, D$ and $F / 2$ to be attained by a smooth curve $c$ as

is the following:

$$
(I I, I, D, F / 2)=(I I(c), I(c), D(c), F(c) / 2)
$$

(i) $I I=I+D+F / 2$, and

(ii) if $F=0, I \leq D^{2}-D$ and $I$ is even. 


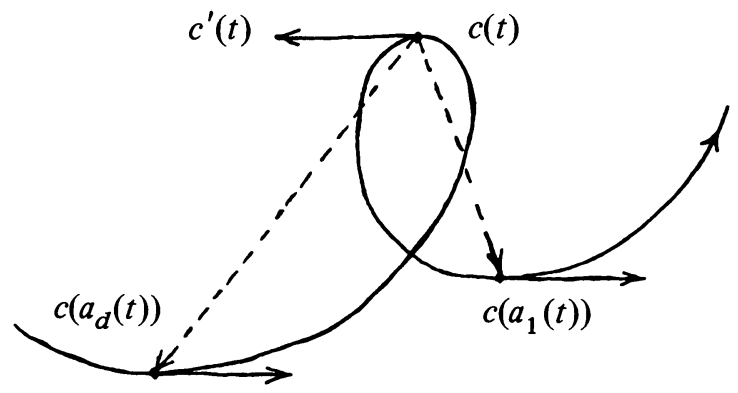

FIGURE 3

In $\S 1$ we prepare some tools for the proof of $I(c) \leq D^{2}(c)-D(c)$, which is done in $\S 2$. In $\S 3$ we construct examples to prove the sufficiency part of the Theorem.

1. In the following, $c$ denotes a smooth closed plane curve parameterized by arc length with only transversal self-intersection and positively curved; $c^{\prime \prime}(t)=$ $d^{2} c(t) / d t^{2}>0$, at every point $t \in S^{1}$, makes sense by identifying the unit circle in $\mathbf{R}^{2}$ and $\mathbf{R} / 2 \pi \mathbf{Z}$ in the usual way.

Since $c^{\prime}=d c / d t: S^{1} \rightarrow S^{1}$ is a covering map, the number of points $t$ in $S^{1}$ such that $c^{\prime}(t)=z$, for a fixed unit vector $z \in S^{1}$, is equal to the tangential degree $d=\operatorname{deg}(c)$. Let $a_{i}: S^{1} \rightarrow S^{1}(i=1, \ldots, d)$ be smooth maps such that for each $t \in S^{1}$,

$$
\left\{\begin{array}{c}
-c^{\prime}(t)=c^{\prime}\left(a_{i}(t)\right) \quad \text { for any } i \\
t<a_{1}(t)<\cdots<a_{d}(t)<t+2 \pi .
\end{array}\right.
$$

Since $c$ has only transversal self-intersection, it follows that $c(t) \neq c\left(a_{i}(t)\right)$ for any $i$ and $t$. Let $g_{i}: S^{1} \rightarrow S^{1}$ denote the functions defined by

$$
g_{i}(t)=\left(c\left(a_{i}(t)\right)-c(t)\right) /\left\|c\left(a_{i}(t)\right)-c(t)\right\|,
$$

and $f_{i}: S^{1} \rightarrow S^{1}$ the functions defined by

$$
f_{i}(t)=c^{\prime}(t)-g_{i}(t)
$$

for $i=1, \ldots, d$, where the symbol "-" in the definition of $f_{i}$ makes sense on the additive group $S^{1}$. We have

$$
\operatorname{deg}\left(f_{i}\right)=\operatorname{deg}\left(c^{\prime}\right)-\operatorname{deg}\left(g_{i}\right)=d-\operatorname{deg}\left(g_{i}\right) .
$$

Each $(t, i)$ with $t \in f_{i}^{-1}(0) \cup f_{i}^{-1}(\pi)$ corresponds to an interior bitangent, and for each interior bitangent there exist two such pairs $(t, i)$ and $(s, j)$. Moreover, for such a pair $(t, i), d g_{i}(t) / d t=0$, and hence $d f_{i}(t) / d t=c^{\prime \prime}(t)>0$. Therefore the number of $t$ 's with $t \in f_{i}^{-1}(0) \cup f_{i}^{-1}(\pi)$ is equal to $2 \operatorname{deg}\left(f_{i}\right)$. Thus we obtain the relation between $\operatorname{deg}\left(f_{i}\right)$ and the number $I(c)$ of the interior bitangents as follows:

LEMMA 1. (i) $\operatorname{deg}\left(f_{i}\right) \geq 0$ for $i=1, \ldots, d$, and (ii) $I(c)=\sum_{i} \operatorname{deg}\left(f_{i}\right)$.

The images $c^{\prime}\left(\left[t, a_{1}(t)\right]\right)$ and $c^{\prime}\left(\left[a_{d}(t), t\right]\right)$ are equal to closed half-circles for each $t$. Hence, from the mean value theorem, it follows that the angles between $c^{\prime}(t)$ and $c\left(a_{1}(t)\right)-c(t)$ and between $c^{\prime}(t)$ and $c\left(a_{d}(t)\right)-c(t)$ are less than $\pi$ for each $t$ (see Figure 3). This implies

LEMMA 2. $0=\operatorname{deg}\left(f_{1}\right)=\operatorname{deg}\left(f_{d}\right)=d-\operatorname{deg}\left(g_{1}\right)=d-\operatorname{deg}\left(g_{d}\right)$. 


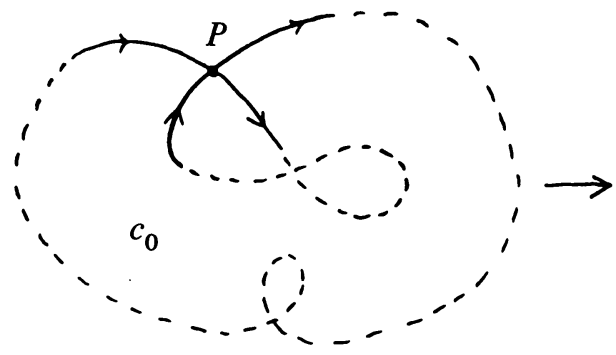

$4 a$.

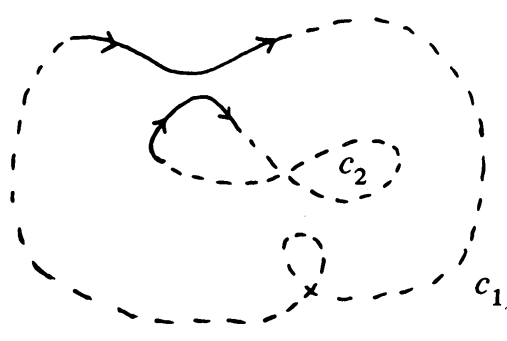

$4 \mathrm{~b}$.

\section{FIGURE 4}

If $c$ has no self-intersection, then $D(c)=0$ and $c$ is a convex curve, since $c^{\prime \prime}(t)>0$ at every point. Hence $\operatorname{deg}(c)=1$. In general we have 1 .

LEMMA 3. If c has no triple points (not necessarily $F(c)=0), D(c) \geq|\operatorname{deg}(c)|-$

PROOF. Suppose there exists a smooth closed curve $c: S^{1} \rightarrow \mathbf{R}^{2}$ such that $D(c)<|\operatorname{deg}(c)|-1$, and let $c_{0}$ be such a curve with the minimal number $D\left(c_{0}\right)$ of self-intersection points. The equality $D(c)=0$ implies that $c$ is a Jordan curve and, hence, $|\operatorname{deg}(c)|=1$. Thus $c_{0}$ has at least one self-intersection point $p$. We replace $c_{0}$ by $c_{1}$ and $c_{2}$ as in Figure $4 \mathrm{~b}$. Then we have

$$
\begin{gathered}
D\left(c_{0}\right) \geq D\left(c_{1}\right)+D\left(c_{2}\right)+1, \\
\left|\operatorname{deg}\left(c_{0}\right)\right|=\left|\operatorname{deg}\left(c_{1}\right)+\operatorname{deg}\left(c_{2}\right)\right| \leq\left|\operatorname{deg}\left(c_{1}\right)\right|+\left|\operatorname{deg}\left(c_{2}\right)\right| .
\end{gathered}
$$

These inequalities and the hypothesis on $c_{0}$ imply

$$
D\left(c_{1}\right)+D\left(c_{2}\right)<\left(\left|\operatorname{deg}\left(c_{1}\right)\right|-1\right)+\left(\left|\operatorname{deg}\left(c_{2}\right)\right|-1\right) .
$$

This and the fact that $D\left(c_{i}\right)<D\left(c_{0}\right)$ for $i=1$ and 2 contradict the minimality of $D\left(c_{0}\right)$. Q.E.D.

In the following, we suppose $c$ has no triple points. We define the subset $A$ of $T^{2}=S^{1} \times S^{1}$ by $A=\left\{(t, s) \in T^{2} ; c(t)=c(s)\right\}$. $A$ is the union of the diagonal set $\Delta \subset T^{2}$ and $2 D(c)$ discrete points which correspond, in pairs, to the self-intersection points of $c$. Let sym: $T^{2} \rightarrow T^{2}$ denote the symmetry of $T^{2} ; \operatorname{sym}(t, s)=(s, t)$. It is clear that $\operatorname{sym}(A)=A$.

To a continuous curve $u=(a, b): S^{1} \rightarrow T^{2}-A$, we associate a map $g_{u}: S^{1} \rightarrow S^{1}$ defined by

$$
g_{u}=(c(b(t))-c(a(t))) /\|c(b(t))-c(a(t))\| .
$$

and define $\operatorname{Deg}(u)=\operatorname{deg}\left(g_{u}\right)$.

LEMMA 4. $\operatorname{Deg}(u)$ is invariant under homotopies, that is, Deg is a function of the homotopy classes of continuous maps of $S^{1}$ to $T^{2}-A$.

PROOF. This is trivial since the degree of $g: S^{1} \rightarrow S^{1}$ is invariant under homotopies. Q.E.D.

We consider the residue $\operatorname{Res}(p)$ of each point $p \in A-\Delta ; \operatorname{Res}(p)=\operatorname{Deg}(u)$, where $u: S^{1} \rightarrow T^{2}-A$ is such that $u$ makes a small round at $p$ positively.

Lemma 5. For each $p \in A-\Delta, \operatorname{Res}(p)= \pm 1$, and $\operatorname{Res}(\operatorname{sym}(p))=-\operatorname{Res}(p)$. 


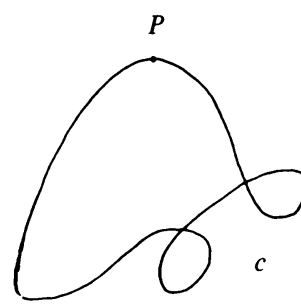

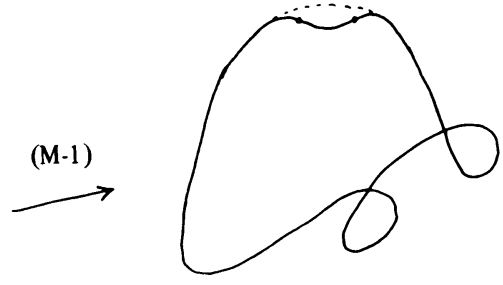

$5 b$.

$5 a$.

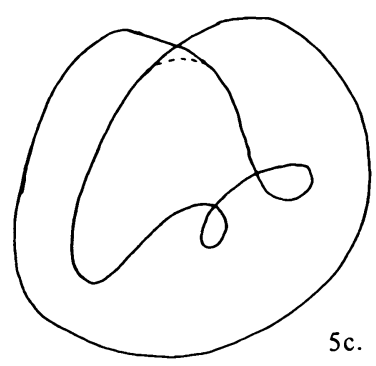

FIGURE 5

ProOF. If we take a continuous imbedding $u=(a, b): S^{1} \rightarrow T^{2}-A$ whose image $u\left(S^{1}\right)$ is a sufficiently small square centered at $p \in A-\Delta$, then we easily find that $g_{u}: S^{1} \rightarrow S^{1}$ is a homeomorphism. Hence, $\operatorname{Res}(p)= \pm 1$.

Let $v$ be obtained from $u$ by parallel translation from $p$ to $\operatorname{sym}(p)$ in $T^{2}$. Then we have $\bar{a}(t)=b(2 \pi-t)$ and $\bar{b}(t)=a(2 \pi-t)$, where $v=(\bar{a}, \bar{b})$, and thus $-g_{v}(t)=g_{u}(-t)$. Hence we have $\operatorname{deg}\left(g_{v}\right)=\operatorname{deg}\left(-g_{v}\right)=-\operatorname{deg}\left(g_{u}\right)$. This implies $\operatorname{Res}(\operatorname{sym}(p))=-\operatorname{Res}(p)$. Q.E.D.

\section{2.}

PROPOSITION (HALPERN'S CONJECTURE). If $F(c)=0$, then $I(c) \leq D^{2}(c)-$ $D(c)$.

ProOF. Suppose $F(c)=0$ and $c^{\prime \prime}>0$ at every point. The proof for $c^{\prime \prime}<0$ is essentially the same. Let $d=\operatorname{deg}(c)$ and let $a_{i}: S^{1} \rightarrow S^{1}(i=1, \ldots, d)$ satisfy (2). Define $u_{i}: S^{1} \rightarrow T^{2}-A$ by $u_{i}(t)=\left(t, a_{i}(t)\right)$. For each $i$ and $j$ with $1 \leq i<j \leq d$, there exists an orientation preserving imbedding $h: S^{1} \times[0,1] \rightarrow T^{2}$ such that $\left.h\right|_{S^{1} \times\{0\}}=u_{i}$ and $\left.h\right|_{S^{1} \times\{1\}}=u_{j}$. Hence, by Lemma 5 we have

$$
\operatorname{Deg}\left(u_{i}\right)-\operatorname{Deg}\left(u_{j}\right)=-\sum_{p \in h\left(S^{1} \times[0,1]\right)} \operatorname{Res}(p)
$$

$\leq$ the number of $p \in A \cap h\left(S^{1} \times[0,1]\right)$ with $\operatorname{Res}(p)=-1$ $\leq D(c)$. 


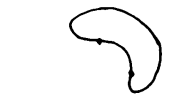

$(I, D, F)=(0,0,2)$

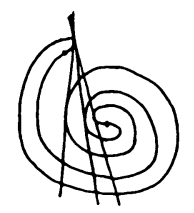

$(I, D, F)=(3,0,2)$

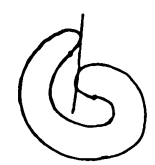

$(I, D, F)=(1,0,2)$

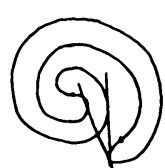

$(I, D, F)=(2,0,2)$

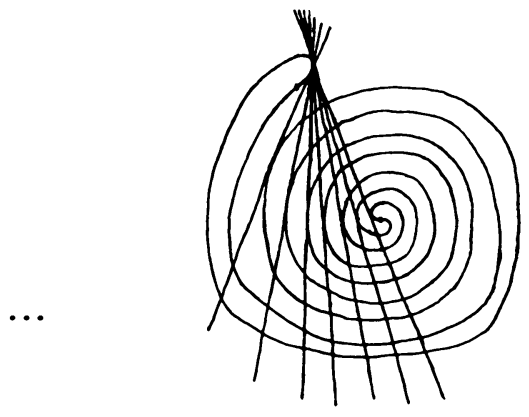

$(I, D, F)=(7,0,2)$

FIGURE 6

By Lemma 2 we have $0=\operatorname{deg}\left(f_{1}\right)=d-\operatorname{Deg}\left(u_{1}\right)$, since $g_{1}=g_{u_{1}}$. Hence, putting $i=1,(4)$ is reduced to

$$
d-\operatorname{Deg}\left(u_{j}\right) \leq D(c)
$$

Thus, from Lemmas 1-3 and inequality (5) it follows that

$$
\begin{aligned}
I(c) & =\sum_{j=1}^{d} \operatorname{deg}\left(f_{j}\right)=\sum_{j=1}^{d}\left(d-\operatorname{Deg}\left(u_{j}\right)\right) \\
& \leq(d-2) D(c) \leq(D(c)-1) D(c) . \quad \text { Q.E.D. }
\end{aligned}
$$

3. Proof of the Theorem (Sufficiency). First, we consider two ways to modify a given curve $c$. Fix a point $p$ on $c$ such that $p$ attains the unique nondegenerate maximum on $c$ of some linear function on $\mathbf{R}^{2}$. In a small neighborhood of $p$, we deform $c$ to $c_{1}$ as in Figure $5 \mathrm{~b}$. Then we have

$$
\left(I\left(c_{1}\right), D\left(c_{1}\right), F\left(c_{1}\right) / 2\right)=(I(c), D(c), F(c) / 2+1) .
$$

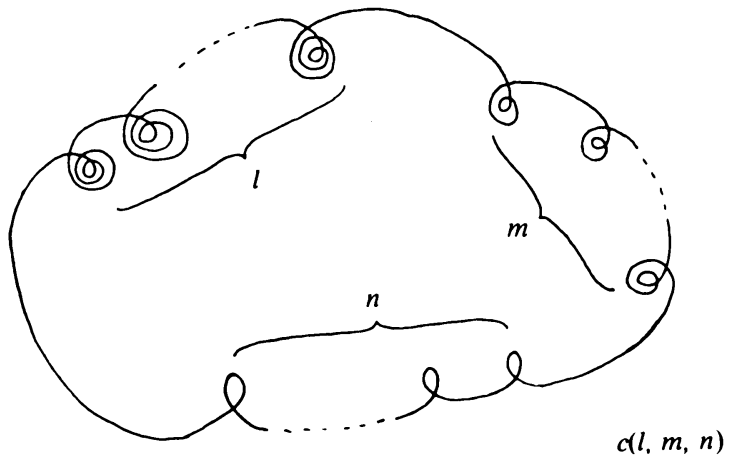

FIGURE 7 
We call this modification (M-1). Next we replace $c$ by $c_{2}$ as in Figure $5 c$. We have

$$
\left(I\left(c_{2}\right), D\left(c_{2}\right), F\left(c_{2}\right) / 2\right)=(I(c), D(c)+1, F(c) / 2) .
$$

This modification is called (M-2).

(i) The case that $c$ has at least two inflection points: For any nonnegative integer $I$, there exists a curve $c$ such that $(I(c), D(c), F(c) / 2)=(I, 0,1)$, as the examples in Figure 6 show. Using the modifications (M-1) and (M-2), it follows that for any nonnegative integers $I, D$ and $F / 2$ with $F / 2 \geq 1$, there exists a curve $c^{\prime}$ such that

$$
\left(I\left(c^{\prime}\right), D\left(c^{\prime}\right), F\left(c^{\prime}\right) / 2\right)=(I, D, F / 2) .
$$

(ii) The case that $c$ has no inflection points: Consider the family of curves $c(l, m, n)$ defined in Figure 7 , where $l, m$ and $n$ are nonnegative integers. The numbers $I, D$ and $F$ of $c(l, m, n)$ are as follows;

$$
\begin{aligned}
F & =F(c(l, m, n))=0, \\
D & =D(c(l, m, n))=3 l+2 m+n, \\
I & =I(c(l, m, n))=D^{2}-D-(6 l+2 m) .
\end{aligned}
$$

We remark that any even integer $x$, with $0 \leq x<2 D-2$, is written as $x=6 l+2 m$ for some nonnegative integers $l$ and $m$ with $3 l+2 m \leq D$. This implies that for given nonnegative integers $D$ and $I$, with

$$
D^{2}-D \geq I>(D-1)^{2}-(D-1)=D^{2}-D-(2 D-2),
$$

there exist $l, m$ and $n$ such that

$$
(I(c(l, m, n)), D(c(l, m, n)), F(c(l, m, n)))=(I, D, 0) .
$$

Combining these examples and (M-2), we finish the proof. Q.E.D.

ACKNOWLEDGEMENT. I wish to thank the referee for indicating to me that Halpern had already proved that the condition in the Theorem of this paper is sufficient, as well as related inequalities, in [4]. Also he indicated to me that Lemma 3 had been proved by Whitney in [5].

\section{REFERENCES}

1. T. Banchoff, Double tangency theorems for pairs of submanifolds, (Geometry Sympos., Utrecht, 1980), Lecture Notes in Math., vol. 894, Springer-Verlag, pp. 26-48.

2. Fr. Fabricius-Bjerre, On the double tangents of plane closed curves, Math. Scand. 11 (1962), 113-116.

3. B. Halpern, Global theorems for closed plane curves, Bull. Amer. Math. Soc. 76 (1970), 96-100.

4. __ An inequality for double tangents, Proc. Amer. Math. Soc. 76 (1979), 133-139.

5. H. Whitney, On regular closed curves in the plane, Compositio Math. 4 (1937), 276-284.

U.E.R. De Mathématiques, Université Paris VII, 75251 PARIS Cedex 05, FranCE

Current address: Department of Mathematics, Faculty of Science, Nagoya University, Chikusaku, Nagoya, 464 Japan 